\title{
Isomer spectroscopy of ${ }^{127} \mathrm{Cd}$
}

F. Naqvi, ${ }^{1,2, *}$ M. Górska, ${ }^{2}$ L. Cáceres,,${ }^{2,3, \dagger}$ A. Jungclaus, ${ }^{4}$ M. Pfützner, ${ }^{5}$ H. Grawe,${ }^{2}$ F. Nowacki,${ }^{6}$ K. Sieja,${ }^{6}$ S. Pietri, ${ }^{7, \ddagger}$ E. Werner-Malento, ${ }^{5,8}$ P. H. Regan, ${ }^{7}$ D. Rudolf, ${ }^{8}$ Z. Podolyák, ${ }^{7}$ J. Jolie, ${ }^{1}$ K. Andgren, ${ }^{9}$ T. Beck, ${ }^{2}$ P. Bednarczyk,, 10 J. Benlliure, ${ }^{11}$ G. Benzoni, ${ }^{12}$ A. M. Bruce, ${ }^{13}$ E. Casarejos, ${ }^{11, \|}$ B. Cederwall, ${ }^{9}$ F. C. L. Crespi,${ }^{12,14}$ P. Detistov, ${ }^{15,}$ Zs. Dombrádi, ${ }^{16}$ P. Doornenbal, ${ }^{2, * *}$ H. Geissel, ${ }^{2}$ J. Gerl, ${ }^{2}$ J. Grębosz, ${ }^{2,10}$ B. Hadinia,${ }^{9}$ M. Hellström ${ }^{8}$ R. Hoischen, ${ }^{2,8}$ G. Ilie, ${ }^{1, \dagger \dagger}$ A. Khaplanov, ${ }^{9}$ I. Kojouharov, ${ }^{2}$ M. Kmiecik, ${ }^{10}$ N. Kurz, ${ }^{2}$ S. Lalkovski,,${ }^{13,15}$ A. Maj, ${ }^{10}$ S. Mandal, ${ }^{17}$ V. Modamio, ${ }^{3}$ F. Montes,, , $+\ddagger$ S. Myalski, ${ }^{10}$ W. Prokopowicz, ${ }^{2}$ P. Reiter, ${ }^{1}$ H. Schaffner, ${ }^{2}$ G. Simpson, ${ }^{18}$ D. Sohler, ${ }^{19}$ S. J. Steer ${ }^{7}$ S. Tashenov, ${ }^{2}$ J. Walker, ${ }^{3}$

O. Wieland, ${ }^{12}$ and H. J. Wollersheim ${ }^{2}$

${ }^{1}$ Institut für Kernphysik, Universität zu Köln, D-50937 Köln, Germany

${ }^{2}$ Helmholtzzentrum für Schwerionenforschung (GSI), D-64291 Darmstadt, Germany

${ }^{3}$ Departmento de Física Teórica, Universidad Autónoma de Madrid, E-28049 Madrid, Spain

${ }^{4}$ Instituto de Estructura de la Materia, CSIC, E-28006 Madrid, Spain

${ }^{5}$ Faculty of Physics, University of Warsaw, PL-00681 Warsaw, Poland

${ }^{6} I P H C$, IN2P3-CNRS/Université Louis Pasteur, F-67037, Strasbourg, France

${ }^{7}$ Department of Physics, University of Surrey, Guildford, GU2 7XH, United Kingdom

${ }^{8}$ Department of Physics, Lund University, S-22100 Lund, Sweden

${ }^{9}$ KTH Stockholm, S-10691 Stockholm, Sweden

${ }^{10}$ The Henryk Niewodniczański Institute of Nuclear Physics, PL-31342 Kraków, Poland

${ }^{11}$ Universidad de Santiago de Compostela, E-175706 Santiago de Compostela, Spain

${ }^{12}$ INFN Sezione di Milano, I-20133 Milano, Italy

${ }^{13}$ School of Engineering, University of Brighton, Brighton, BN2 4GJ, United Kingdom

${ }^{14}$ Universitá degli Studi di Milano, I-20133 Milano, Italy

${ }^{15}$ Faculty of Physics, University of Sofia, BG-1164, Sofia, Bulgaria

${ }^{16}$ Institute National Polytechnique de Grenoble, F-98026 Grenoble Cedex, France

${ }^{17}$ University of Delhi, New Delhi, India

${ }^{18}$ Institut Laue-Langevin, F-38042 Grenoble, France

${ }^{19}$ Institute of Nuclear Research, Pf. 51, H-4001 Debrecen, Hungary

(Received 5 July 2010; published 24 September 2010)

\begin{abstract}
The spin and configurational structure of excited states of ${ }^{127} \mathrm{Cd}$, the two-proton and three-neutron hole neighbor of ${ }^{132} \mathrm{Sn}$, has been studied. An isomeric state with a half-life of $17.5(3) \mu$ s was populated in the fragmentation of a ${ }^{136} \mathrm{Xe}$ beam on a ${ }^{9} \mathrm{Be}$ target at a beam energy of $750 \mathrm{MeV} / \mathrm{u}$. Time distributions of the delayed $\gamma$ transitions and $\gamma \gamma$ coincidence relations were exploited to construct a decay scheme. The observed yrast $(19 / 2)^{+}$isomer is proposed to have dominant configurations of $v\left(h_{11 / 2}^{-3}\right) \pi\left(g_{9 / 2}^{-1}, p_{1 / 2}^{-1}\right), v\left(h_{11 / 2}^{-2} d_{3 / 2}^{-1}\right) \pi\left(g_{9 / 2}^{-2}\right)$, and $v\left(h_{11 / 2}^{-2}, s_{1 / 2}^{-1}\right) \pi\left(g_{9 / 2}^{-2}\right)$ and to decay by two competing stretched $M 2$ and $E 3$ transitions. Experimental results are compared with the isotone ${ }^{129} \mathrm{Sn}$. The new information provides input for the proton-neutron interaction and the evolution of neutron hole energies in nuclei around the doubly magic ${ }^{132} \mathrm{Sn}$ core.
\end{abstract}

DOI: 10.1103/PhysRevC.82.034323

PACS number(s): 21.10.Tg, 23.20.Lv, 23.35.+g, 27.60.+j

\section{INTRODUCTION}

The region around doubly magic ${ }^{132} \mathrm{Sn}$ has been a subject of intense research because of its several interesting features. Few valence particles and holes outside the inert core make these nuclei key points in studying the shell structure and

\footnotetext{
*F.Naqvi@gsi.de

${ }^{\dagger}$ Present address: GANIL Caen, France.

${ }^{\ddagger}$ Present address: GSI Darmstadt, Germany.

${ }^{\S}$ Present address: IF PAN, Warsaw, Poland.

"Present address: University of Vigo, Spain.

Present address: Universidad de Salamanca, Spain.

*** Present address: RIKEN, Japan.

${ }^{\dagger}$ Present address: Yale University, USA.

${ }_{\ddagger}^{\ddagger}$ Present address: University of Michigan, USA.
}

nucleon-nucleon interaction in a neutron-rich environment. The astrophysical rapid-neutron capture process, responsible for the synthesis of heavy neutron-rich nuclei in nature, follows a path along the $N=82$ line up to $Z=48$. To test the theoretical models describing this process in the region around $A=130$, the availability of experimental data on the evolution of the $N=82$ shell gap is crucial. The predicted reduction of this gap [1,2] led to the recent investigations on the structure of ${ }^{130} \mathrm{Cd}$ [3] and ${ }^{131} \mathrm{In}$ [4]. The energy of the first excited $2^{+}$state in ${ }^{130} \mathrm{Cd}$ indirectly points toward a robust shell closure at $N=82$, which was confirmed by a direct measure of the shell gap in Ref. [4]. Missing evidence for a shell quenching in all these cases calls for a need to modify the mass model [5] explaining the abundances of $A=130$ nuclei in nature. Furthermore, information on the structure of excited states in ${ }^{126} \mathrm{Cd}$ and ${ }^{128} \mathrm{Cd}[6,7]$ indicates an enhanced quadrupole collectivity, which has also been 

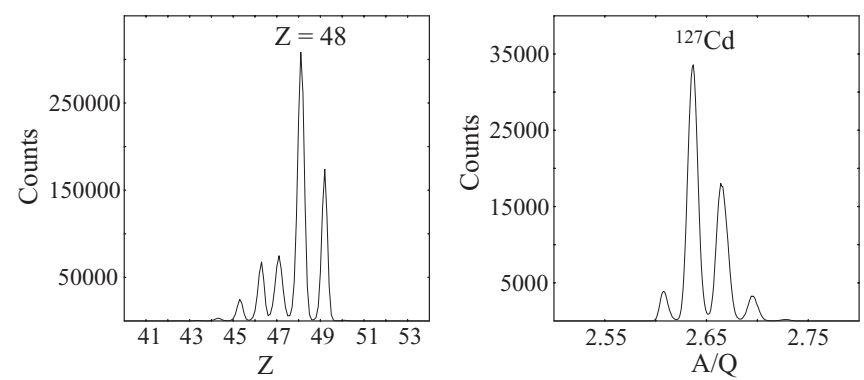

FIG. 1. Left: $Z$ information provided by the two ionization chambers. Right: $A / Q$ selection for Cd isotopes.

revealed in theoretical studies [8]. To gain more insight on this issue and to extend the study of structure evolution in the $\mathrm{Cd}$ chain to the odd isotopes, a detailed examination of the data from an experimental campaign performed at the FRagment Separator (FRS) [9] at GSI was done. A level scheme for ${ }^{127} \mathrm{Cd}$ supported by the detailed experimental information including coincidence relations and isomeric half-life has been constructed for the first time in the present work.

\section{EXPERIMENTAL TECHNIQUE}

Isomeric decays of ${ }^{127} \mathrm{Cd}$ were observed following the relativistic fragmentation reaction of ${ }^{136} \mathrm{Xe}$ beam at an energy of $750 \mathrm{MeV} / \mathrm{u}$ on a ${ }^{9} \mathrm{Be}$ target of $4 \mathrm{~g} / \mathrm{cm}^{2}$ thickness. The neutron-rich $\mathrm{Cd}$ isotopes were produced in two different settings of the FRS, one optimized for the maximum transmission of ${ }^{130} \mathrm{Cd}$ and the other one tuned to predominantly transmit ${ }^{126} \mathrm{Cd}$. To select the ions of interest from the reaction products the standard $B \rho-\Delta E-B \rho$ method was employed and the FRS was operated in its achromatic mode. Event-by-event identification of the particles in terms of their mass $(A)$ and charge $(Z)$ was done by measuring the time of flight between two scintillator detectors separated by a distance of $35 \mathrm{~m}$ and the magnetic rigidities providing the $A / Q$ ratio. Energy loss information in two ionization chambers served to determine the atomic number $Z$. Corresponding plots of $Z$ and $A / Q$ identification are shown in Fig. 1. Isomers populated in the reaction, having lifetimes long enough to be able to reach the final focal plane, were implanted in a plastic catcher, surrounded by 15 Ge cluster detectors from the RISING array, arranged in close geometry [10]. Coincidence between the implanted ${ }^{127} \mathrm{Cd}$ ions and the detected $\gamma$ rays within a time range of $50 \mu$ s ensured an unambiguous assignment of the detected radiations to the decay of the isomeric states of the ions. Stringent conditions imposed on the selection procedure of the FRS along with a clean identification of the particles enabled the observation of $7.2 \times 10^{5}{ }^{127} \mathrm{Cd}$ ions in total from the two settings.

\section{EXPERIMENTAL RESULTS}

A singles $\gamma$-ray spectrum containing five $\gamma$ transitions assigned to the decay of the isomer in ${ }^{127} \mathrm{Cd}$ is shown in Fig. 2. So far $\gamma$ transitions in ${ }^{127} \mathrm{Cd}$ were identified in two

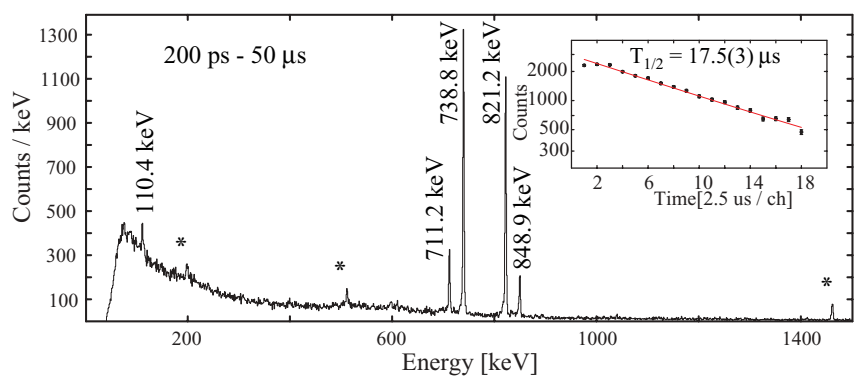

FIG. 2. (Color online) Singles $\gamma$-ray energy spectrum of ${ }^{127} \mathrm{Cd}$. Background lines are marked with *. Inset: The sum of time distributions of the 711-, 739-, 821-, and 849-keV transitions with a single exponential decay fit.

previous experiments. Hellström et al. [11] assigned four $\gamma$ transitions at energies of $710,738,820$, and $342 \mathrm{keV}$ to the isomeric decay. In the work of Hoteling et al. [12], apart from the two previously observed $738-$ and $820-\mathrm{keV} \gamma$ rays, two new $\gamma$ transitions at energies of 770.9 and $908.0 \mathrm{keV}$ were identified. Though a level scheme for ${ }^{127} \mathrm{Cd}$ was constructed only in the latter paper, it was merely based on the systematics of known neighboring odd-mass $\mathrm{Cd}$ isotopes owing to low statistics and lack of lifetime information. In our experiment the previously reported $\gamma$ transitions at energies of 739, 821, and $711 \mathrm{keV}$ were affirmed and two new $\gamma$ rays at energies of 110 and $849 \mathrm{keV}$ were identified. However, the 771- and 909-keV transitions reported in Ref. [12] and the 342-keV transition reported in Ref. [11] could not be confirmed in the present work. The newly observed $\gamma \gamma$ coincidence relations of the five transitions associated with the isomeric decay in ${ }^{127} \mathrm{Cd}$ are shown in Fig. 3. Coincidences were investigated for two different coincidence time windows of $100 \mathrm{~ns}$ and $50 \mu \mathrm{s}$.

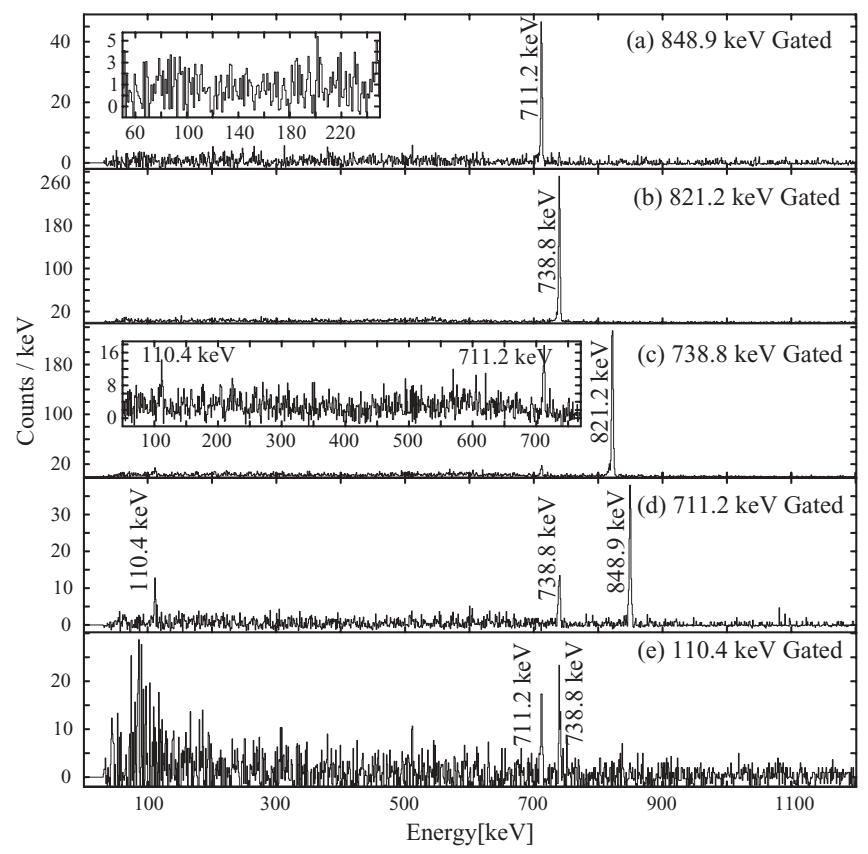

FIG. 3. $\gamma \gamma$ coincidence spectra with a coincidence window of 100 ns. Inset: Zoomed-in region of the respective weak coincidences. 
TABLE I. Summary of the measured half-lives and spin and parity assignments of the levels in ${ }^{127} \mathrm{Cd}$. Energy of the states are with respect to the $\left(11 / 2^{-}\right) \beta^{-}$decaying isomer. The $\gamma$ intensities are normalized to the $739-\mathrm{keV}$ transition.

\begin{tabular}{|c|c|c|c|c|c|}
\hline $\begin{array}{l}E_{i}+x \\
(\mathrm{keV})\end{array}$ & $\begin{array}{l}T_{1 / 2} \\
(\mu \mathrm{s})\end{array}$ & $\begin{array}{c}\text { Transition } \\
\quad J \rightarrow I\end{array}$ & $\begin{array}{c}E_{\gamma} \\
(\mathrm{keV})\end{array}$ & $\begin{array}{l}T_{1 / 2} \\
(\mu \mathrm{s})\end{array}$ & $\begin{array}{l}I_{\gamma} \\
(\%)\end{array}$ \\
\hline 711 & & $\left(13 / 2^{-}\right) \rightarrow\left(11 / 2^{-}\right)$ & $711.2(2)$ & $17.1(8)^{\mathrm{b}}$ & $23.0(9)$ \\
\hline \multirow[t]{2}{*}{821} & & $\left(15 / 2^{-}\right) \rightarrow\left(11 / 2^{-}\right)$ & $821.2(2)$ & $17.7(4)^{\mathrm{b}}$ & $91.8(30)$ \\
\hline & & $\left(15 / 2^{-}\right) \rightarrow\left(13 / 2^{-}\right)$ & $110.4(2)$ & $17.5(12)^{b}$ & $6.2(6)$ \\
\hline \multirow[t]{2}{*}{1560} & $17.5(3)^{\mathrm{a}}$ & $\left(19 / 2^{+}\right) \rightarrow\left(15 / 2^{-}\right)$ & $738.8(2)$ & $17.6(4)^{b}$ & $100.0(31)$ \\
\hline & & $\left(19 / 2^{+}\right) \rightarrow\left(13 / 2^{-}\right)$ & $848.9(2)$ & $17.9(6)^{b}$ & $17.3(7)$ \\
\hline
\end{tabular}

${ }^{\text {aExtracted from the fitting of the sum of time distributions of the 711-, 739-, 821-, and }}$ 849-keV transitions with a single exponential decay function.

${ }^{b}$ Extracted from the fitting of the time distribution of corresponding individual $\gamma$ transition with a single exponential decay function.

Comparable relative intensities of all the $\gamma$ transitions in the aforementioned conditions established the presence of only one isomer in the decay.

The proposed level scheme for ${ }^{127} \mathrm{Cd}$ is based on the following arguments. The two most intense $\gamma$ transitions of 739 and $821 \mathrm{keV}$ most likely form a single cascade, which is confirmed by their mutual coincidence shown in Fig. 3(b). The 711- and 849-keV lines have similar intensities and are in coincidence [see Fig. 3(a)], which points toward the existence of a second cascade in the decay. The sum of energies of the 739- and $821-\mathrm{keV}$ lines is equal to that of the 849- and 711-keV lines. Also, the absence of coincidence between these two cascades confirms that they are parallel competing branches. Observation of a 110-keV transition coinciding with the differences in energies of the 849- and 739-keV lines and the 711- and 821-keV lines suggests that an intermediate level is present, fed by the $110-\mathrm{keV}$ transition. Coincidence between $110-\mathrm{keV}$ and $711-$ or $739-\mathrm{keV}$ lines supports the argument of an intermediate level connecting the two cascades via the $110-\mathrm{keV}$ transition. The lifetime analysis based on the single exponential decay fit of the summed time distributions of the 711-, 739-, 821-, and 849-keV transitions is shown in the inset of Fig. 2. A half-life of 17.5(3) $\mu$ s was obtained for the decaying isomer. The fit of the time distributions of individual $\gamma$ lines with the same function as described here was also performed and the results are listed in Table I. Because

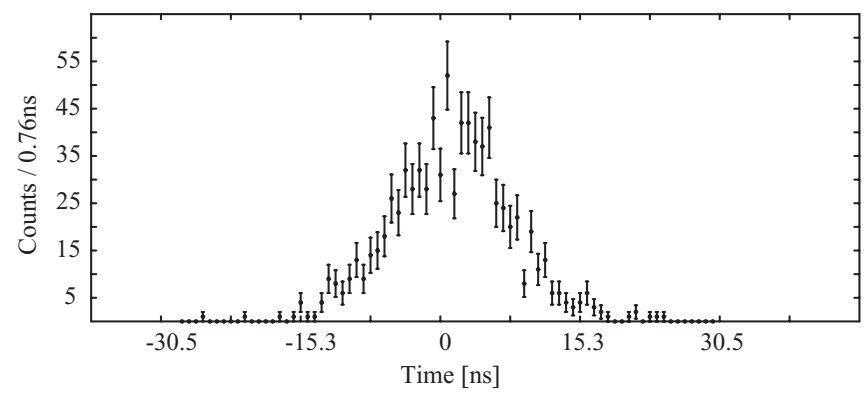

FIG. 4. Relative time distribution of the $739-\mathrm{keV}$ transition with respect to the $821-\mathrm{keV}$ transition. The spectrum shows a symmetric distribution. the $110-\mathrm{keV}$ transition has a relative intensity of only $6 \%$, the presence of a $115-\mathrm{keV}$ background line in its vicinity made the background subtraction difficult. This resulted in a comparatively larger error in the measured value.

From the half-life information and the inferred reduced transition probabilities, the multipolarity of the $\gamma$ transitions is determined. Owing to similar energies and same half-lives of 711-, 739-, 821-, and 849-keV lines, all four of them qualify to be either $M 2$ or $E 3$, if one assumes that the isomerism is caused by any of them. Hence the ordering of these transitions could not be determined based on the experimental data. The relative time between the 739 - and $821-\mathrm{keV}$ transitions shows a symmetric distribution (see Fig. 4) within an electronic resolution of $0.7 \mathrm{~ns}$. This implies that the state depopulated by the $110-\mathrm{keV}$ line in competition with the $821-$ or $739-\mathrm{keV}$ transitions has a half-life of less than $10 \mathrm{~ns}$ since a longer lifetime would lead to clear asymmetries as observed in the case of ${ }^{128} \mathrm{Cd}$ [7]. Only an $M 1$ character of the $110 \mathrm{keV}$ transition would be compatible with this upper half-life limit.

\section{DISCUSSION}

Spins and parities were assigned to the excited states in ${ }^{127} \mathrm{Cd}$ on the basis of intensity balances, reduced transition probabilities, and the experimental information on the level scheme of the ${ }^{129} \mathrm{Sn}$ isotone [13]. According to the $\beta^{-}$decay studies of ${ }^{127} \mathrm{Cd},\left(3 / 2^{+}\right)$is assigned to the $\beta^{-}$decaying ground state [14] with pure $v d_{3 / 2}$ neutron character. The systematics of odd-mass $\mathrm{Cd}$ and $\mathrm{Sn}$ isotopes [12,13] confirms that the $\gamma$-decaying isomer is built on a $\left(11 / 2^{-}\right)$state. Owing to the large spin difference and long partial lifetime, $\gamma$ transition between the $\left(11 / 2^{-}\right)$and $\left(3 / 2^{+}\right)$states is not observed and thus the former decays by a $\beta^{-}$emission. From the existing experimental evidence it cannot be firmly established whether the $\left(3 / 2^{+}\right)$or the $\left(11 / 2^{-}\right)$state is the ground state. ${ }^{129} \mathrm{Sn}$ having the same number of neutron holes in the ${ }^{132} \mathrm{Sn}$ core as ${ }^{127} \mathrm{Cd}$ provides the best case for comparison. Since in ${ }^{129} \mathrm{Sn}$ isomerism is mainly caused by $E 2$ transitions of very low energies or a hindered $M 2\left(19 / 2^{+}\right) \rightarrow\left(15 / 2^{-}\right)$transition, a similar explanation was assumed to be the reason of isomer formation in ${ }^{127} \mathrm{Cd}$ by Hellström et al. [11]. 
In ${ }^{127} \mathrm{Cd} \mathrm{a}\left(19 / 2^{+}\right) \rightarrow\left(15 / 2^{+}\right)$E2 spin trap analogous to the one observed in ${ }^{129} \mathrm{Sn}$ given the half-life of $T_{1 / 2}=$ $17.5 \mu$ s would require a nonobserved transition of $\sim 30 \mathrm{keV}$ and the observation of two competing $E 1$ transitions, $\left(15 / 2^{+}\right) \rightarrow\left(15 / 2^{-}\right)$and $\left(15 / 2^{+}\right) \rightarrow\left(13 / 2^{-}\right)$, with similar intensities. But the encountered branching ratios of $849 \mathrm{keV}$ with respect to $739 \mathrm{keV}$ and of $711 \mathrm{keV}$ with respect to $821 \mathrm{keV}$ rules out this possibility. Alternatively, if a $15 / 2^{+}$state happens to lie above the $19 / 2^{+}$state causing an isomer, the fast $15 / 2^{+} \rightarrow 15 / 2^{-}$and $15 / 2^{+} \rightarrow 13 / 2^{-}$transitions would not let the corresponding state be isomeric with a half-life of $17.5 \mu \mathrm{s}$. Hence we can conclude that an $M 2$ transition from $\left(19 / 2^{+}\right)$to $\left(15 / 2^{-}\right)$in competition with an $E 3$ transition from $\left(19 / 2^{+}\right)$to $\left(13 / 2^{-}\right)$is responsible for the measured half-life of the isomer. This argument allowed us to assign a spin of $\left(19 / 2^{+}\right)$to the decaying isomeric state. The decay of the intermediate state is governed by a competition between the 110- and 821- or 849-keV transitions. The experimental branching ratios for the $110-\mathrm{keV} \gamma$ line in the two cases are $\sim 6 \%$ and $26 \%$, respectively, while the expected value from the energy scaling of two such $M 1$ transitions of equal reduced strength is $\sim 0.5 \%$. Therefore, this intermediate state was assigned to have spin-parity $\left(15 / 2^{-}\right)$, depopulated by a competition of a $110-\mathrm{keV} M 1$ transition and a $821-$ or $849-\mathrm{keV}$ $E 2$ transition. Owing to the uncertainty in the ordering of $\gamma$ rays just discussed the intermediate states at 711 and $821 \mathrm{keV}$ shown in the level scheme in Fig. 5 (EXP) could lie at 739 and $849 \mathrm{keV}$ instead with respect to the $\left(11 / 2^{-}\right)$state. So two possibilities for the experimental level scheme of ${ }^{127} \mathrm{Cd}$ are proposed, one shown in Fig. 5 and the other with reversed ordering of the 739- and 849-keV transitions and the 821- and 711-keV transitions.

\section{A. Shell-model interpretation}

Two theoretical shell-model approaches employing different model spaces and interactions were used to investigate the nuclear structure evidence of the experimental data. In the first one a model space $\pi\left(p_{1 / 2}, g_{9 / 2}\right) v\left(d_{5 / 2}, g_{7 / 2}, s_{1 / 2}, d_{3 / 2}, h_{11 / 2}\right)$ outside a ${ }^{88} \mathrm{Sr}$ core was chosen (i.e., core excitations across the $Z=50$ and $N=82$ shells are not considered). The

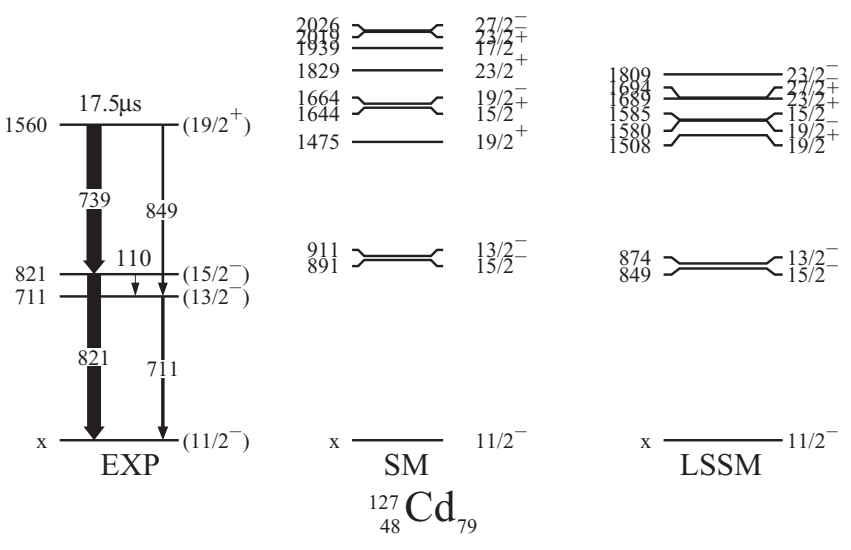

FIG. 5. The proposed level scheme for ${ }^{127} \mathrm{Cd}$ and the theoretically calculated excited levels by SM and LSSM. residual interaction is based on a $G$ matrix derived from the CD-Bonn nucleon-nucleon potential [15] and core polarization corrections for a ${ }^{88} \mathrm{Sr}$ inert core following the many-body approach outlined in Ref. [16]. Monopole tuning following $A^{-1 / 3}$ scaling of the two-body matrix elements (TBME) was performed to reproduce the single hole energies (SHE) in ${ }^{132} \mathrm{Sn}$ as described in Refs. [3,13]. For the open proton-neutron $(\pi v)$ space two modifications were introduced. First the underbound $\pi g_{9 / 2} v h_{11 / 2}, I^{\pi}=1^{-}, 10^{-}$TBME were increased by -500 and $-600 \mathrm{keV}$, respectively, keeping the multiplet monopole constant. This is necessary to reproduce the ground and isomeric states in ${ }^{130}$ In [14]. Second, to increase the collectivity in the $(\pi v)$ shells, the dominating binding (negative) diagonal $\pi v$ TBME $\pi g_{9 / 2} v h_{11 / 2}$ and $\pi p_{1 / 2} v h_{11 / 2}$ were enhanced by a monopole shift of $-100 \mathrm{keV}$. To maintain the SHE for ${ }^{132} \mathrm{Sn}$, the experimentally unknown $v h_{11 / 2} \mathrm{SPE}$ in ${ }^{88} \mathrm{Sr}$ was increased by $+1.2 \mathrm{MeV}$. Further the $\left(v h_{11 / 2}\right)_{2+}^{2}$ TBME was reduced by $-150 \mathrm{keV}$, keeping the monopole of the multiplet constant, to improve the agreement in the even Sn isotopes. With these modifications, referred to as SM, the agreement with experiment in ${ }^{128} \mathrm{Cd}$ [17] and ${ }^{127} \mathrm{Cd}$ is greatly improved, though the $\left(13 / 2^{-}\right)-\left(15 / 2^{-}\right)$ sequence in the latter is not fully reproduced (Fig. 5). Further details will be given in a forthcoming paper [17]. Effective charges $e_{\pi}=1.5 e$ and $e_{\nu}=0.7 e$ for protons and neutrons and a spin $g$ factor of $g_{s}=0.7 g_{s}^{\text {free }}$ for magnetic transitions were used [3,13]. Calculations were performed with the code OXBASH [18]. In the second approach an extended model space $\pi\left(p_{3 / 2}, p_{1 / 2}, f_{5 / 2}, g_{9 / 2}\right) v\left(d_{5 / 2}, g_{7 / 2}, s_{1 / 2}, d_{3 / 2}, h_{11 / 2}\right)$ outside ${ }^{78} \mathrm{Ni}$ was chosen with an interaction derived from the CD-Bonn nucleon-nucleon potential using the same method as just described, but for an inert ${ }^{78} \mathrm{Ni}$ core [7]. Monopole tuning was performed to reproduce the experimental SHE for ${ }^{132} \mathrm{Sn}$ and levels in neighboring $\mathrm{Sn}$ isotopes, the $N=82$ isotone ${ }^{130} \mathrm{Cd}$, and the $\pi^{-1} v^{-1}$ neighbor ${ }^{130} \mathrm{In}$. Effective charges of $1.5 e$ for protons and $0.5 e$ for neutrons were used for electric transitions and an effective spin $g$ factor of $g_{s}=0.75 g_{s}$ free for magnetic transitions. In addition orbital $g$ factors of $g_{l}(p)=1.1$ and $g_{l}(n)=-0.1$ for protons and neutrons, respectively were used. Calculations were performed with the large-scale shell-model (LSSM) codes ANTOINE and NATHAN [19] and the results are denoted by LSSM in Fig. 5.

From the good agreement of the shell-model approaches SM and LSSM for the experimentally known levels it can be concluded that the increased $\pi v$ interaction in SM to a large extent accounts for the neglect of the $\pi\left(p_{3 / 2}, f_{5 / 2}\right)$ orbitals in the model space used. The $2^{+}$excitation energy in ${ }^{128} \mathrm{Cd}$ is still high by $\sim 150 \mathrm{keV}$. This discrepancy is carried over into the energy of $\left(13 / 2^{-}\right)$state in ${ }^{127} \mathrm{Cd}$, which has leading configuration of a $v h_{11 / 2}$ hole coupled to the $2_{1}^{+}$state in ${ }^{128} \mathrm{Cd}$. These remaining deficiencies, with the high-spin isomers being well reproduced, indicate a lack of collectivity in the low-spin states. Lifting the $\pi\left(p_{3 / 2}, f_{5 / 2}\right)$ orbitals closer to the Fermi surface would create a pseudo-SU(3) $h h$ space comprising the $\pi\left(p, f_{5 / 2}\right)$ and $v(s, d)$ orbitals. This would further increase collectivity as observed one major shell higher for the $\mathrm{Hg}$ isotopes in the ${ }^{208} \mathrm{~Pb} h h$ space as in the well studied $(s, d)$ and $(p, f) p p$ cases (see, e.g., [20]). Reduced transition probabilities in the two experimental scenarios 
TABLE II. Transition strengths and $\gamma$ branching ratios for experiment and shell model. Separate values are given for the two alternative experimental level schemes $\mathrm{EXP}^{\mathrm{a}}$ and $\mathrm{EXP}^{\mathrm{b}}$ of ${ }^{127} \mathrm{Cd}$.

\begin{tabular}{|c|c|c|c|c|c|}
\hline \multirow[t]{2}{*}{ Transition } & \multirow{2}{*}{$\begin{array}{c}\sigma \mathrm{L} \\
J \rightarrow I\end{array}$} & \multicolumn{4}{|c|}{$B(\sigma \mathrm{L})(\mathrm{W} . \mathrm{u})$} \\
\hline & & $\mathrm{EXP}^{\mathrm{a}}$ & $\mathrm{EXP}^{\mathrm{b}}$ & SM & LSSM \\
\hline$\left(19 / 2^{+}\right) \rightarrow\left(13 / 2^{-}\right)$ & $E 3^{\mathrm{c}}$ & $3.40(18) \times 10^{-2}$ & $2.30(30) \times 10^{-1}$ & $6.19 \times 10^{-3}$ & $3.04 \times 10^{-3}$ \\
\hline$\left(19 / 2^{+}\right) \rightarrow\left(15 / 2^{-}\right)$ & $M 2^{\mathrm{c}}$ & $2.71(14) \times 10^{-4}$ & $7.71(16) \times 10^{-5}$ & $1.98 \times 10^{-3}$ & $5.75 \times 10^{-3}$ \\
\hline$\left(15 / 2^{-}\right) \rightarrow\left(13 / 2^{-}\right)$ & $M 1^{\mathrm{d}}$ & $6.3(6) \%$ & $26(3) \%$ & $5.8 \%$ & $7.5 \%$ \\
\hline$\left(15 / 2^{-}\right) \rightarrow\left(11 / 2^{-}\right)$ & $E 2^{\mathrm{d}}$ & $93.7(31) \%$ & $74(3) \%$ & $94.2 \%$ & $92.5 \%$ \\
\hline
\end{tabular}

\footnotetext{
${ }^{a}$ Adopted order of cascades.

${ }^{\mathrm{b}}$ Reversed order of cascades.

${ }^{\mathrm{c}}$ Pure multipolarity assumed.

${ }^{\mathrm{d} O n l y} \gamma$ branching ratio known.
}

are compared to SM and LSSM results in Table II. The retarded $B\left[E 3 ;\left(19 / 2^{+}\right) \rightarrow\left(13 / 2^{-}\right)\right]$and $B\left[M 2 ;\left(19 / 2^{+}\right) \rightarrow\right.$ $\left.\left(15 / 2^{-}\right)\right]$are, respectively, under- and overestimated for both experimental scenarios. In the model space these transitions are $v h_{11 / 2} \rightarrow d_{5 / 2}$ and $v h_{11 / 2} \rightarrow g_{7 / 2}$, which include small components in the wave function that may be distorted by configurations from outside the model space. Moreover, the effective charges and $g$ factors for $E 3$ and $M 2$ operators may be different from those for $E 2$ and $M 1$, respectively. A good correspondence between experimental and theoretical branching ratio is observed for the $\left(15 / 2^{-}\right) \rightarrow\left(11 / 2^{-}\right) 821$ $\mathrm{keV} E 2$ and the $\left(15 / 2^{-}\right) \rightarrow\left(13 / 2^{-}\right) 110-\mathrm{keV} M 1$ transitions in the scenario shown in Fig. 5. It should be noted that the SM and LSSM values agree as well. The drastic discrepancy for this branching in the alternative cascade assignment and slightly better agreement for the $M 2$ and $E 3$ transitions (see Table II) establishes a preference for the adopted order.

\section{SUMMARY AND CONCLUSIONS}

In conclusion, a microsecond isomer has been identified in ${ }^{127} \mathrm{Cd}$. Based on $\gamma \gamma$ coincidence and lifetime analysis a level scheme on top of a $\nu h_{11 / 2}^{-1}$ isomer or ground state was constructed for this nucleus. In contrast to the odd-mass heavy $\mathrm{Sn}$ isotopes the isomerism in ${ }^{127} \mathrm{Cd}$ is attributed to competing hindered $E 3$ and $M 2$ transitions from a $\left(19 / 2^{+}\right)$state, formed by mixed proton-neutron configurations. The sequence of the intermediate $\left(13 / 2^{-}\right)$and $\left(15 / 2^{-}\right)$states serves as a benchmark for shell-model calculations performed with two different interactions in a small (SM) and an extended (LSSM) model space. Since the theoretical models reproduce the energies of the low-lying pure proton and pure neutron excited levels in ${ }^{130} \mathrm{Cd}$ and ${ }^{130} \mathrm{Sn}$, respectively, an enhanced proton-neutron interaction is attributed to be the origin of increased collectivity in the low-spin yrast region of ${ }^{127} \mathrm{Cd}$.

\section{ACKNOWLEDGMENTS}

The authors would like to acknowledge the support of German Federal Ministry of Education and Research (06KY205I), the EU Access to Large Scale Facilities Programme (EURONS, EU Contract No. 506065), the Polish Ministry of Science and Higher Education (1-P03B-030-30 and 620/E-77/SPB/GSI/P-03/DWM105/2004-2007), the Spanish Ministerio de Ciencia e Innovación (FPA2007-66069 and FPA2009-13377-C02-02), EPSRC/STFC (UK), the Swedish Research Council, the Bulgarian Science Fund VUF06/05, the US Department of Energy (DE-FG02-91ER-40609 and DE-AC02-06CH11357), the Hungarian Science Foundation (OTKA K-68801), and the Italian INFN.
[1] J. Dobaczewski, I. Hamamoto, W. Nazarewicz, and J. A. Sheikh, Phys. Rev. Lett. 72, 981 (1994).

[2] I. Dillmann et al., Phys. Rev. Lett. 91, 162503 (2003).

[3] A. Jungclaus et al., Phys. Rev. Lett. 99, 132501 (2007).

[4] M. Górska et al., Phys. Lett. B 672, 313 (2009).

[5] B. Chen et al., Phys. Lett. B 355, 37 (1995).

[6] T. Kautzsch et al., Eur. Phys. J. A 9, 201 (2000).

[7] L. Cáceres et al., Phys. Rev. C 79, 011301(R) (2009).

[8] T. R. Rodríguez, J. L. Egido, and A. Jungclaus, Phys. Lett. B 668, 410 (2008).

[9] H. Geissel et al., Nucl. Instrum. Methods Phys. Res. B 70, 286 (1992).

[10] S. Pietri et al., Nucl. Instrum. Methods Phys. Res. B 261, 1079 (2007).
[11] M. Hellström et al., GSI 2003-1, 2003, p. 5 (unpublished).

[12] N. Hoteling et al., Phys. Rev. C 76, 044324 (2007).

[13] R. Lozeva et al., Phys. Rev. C 77, 064313 (2008).

[14] ENSDF database, [http://www.nndc.bnl.gov/ensdf/].

[15] R. Machleidt, Phys. Rev. C 63, 024001 (2001).

[16] M. Hjorth-Jensen, T. T. S. Kuo, and E. Osnes, Phys. Rep. 261, 125 (1995).

[17] H. Grawe et al. (to be published).

[18] B. A. Brown et al., OXBASH for Windows, MSU-NSCL Report 1289, 2004.

[19] E. Caurier, G. Martínez-Pinedo, F. Nowacki, A. Poves and A. P. Zuker, Rev. Mod. Phys. 77, 427 (2005).

[20] R. F. Casten et al., Algebraic Approaches to Nuclear Structure:Interacting Boson and Fermion Model (Harwood Academic Publishers, 1993). 